\title{
Programa Articulado Nutricional y su impacto en la desnutrición crónica en menores de cinco años Dirección Regional de Salud Callao, 2009-2015
}

Keila Shajian-Torres* 1,a; José Garay-Uribe 1, b; Ricardo Carreño-Escobedo 1,c

RESUMEN

Objetivo: Determinar el impacto del Programa Articulado Nutricional (PAN) en la desnutrición crónica en menores de cinco años, su correlación con la ejecución presupuestal del PAN y la prevalencia de la desnutrición crónica en la Dirección Regional de Salud (DIRESA) Callao, 2009-2015.

Materiales y métodos: Estudio cuanticualitativo, observacional ecológico, correlacional, longitudinal y retrospectivo. Se determinó el coeficiente de correlación de Pearson en el análisis cuantitativo. Los datos cualitativos se obtuvieron a partir de entrevistas a profundidad con apoyo de una guía semiestructurada.

Resultados: No existe una relación entre el Presupuesto Institucional Modificado (PIM) del PAN y la prevalencia de desnutrición crónica $(p=0,92)$. No existe una relación entre la ejecución presupuestal del PIM y la prevalencia de desnutrición crónica $(p=0,355)$. Los factores que influyeron en la ejecución del PAN son presupuesto inoportuno, retraso en la implementación del PAN, dificultades en la organización de los servicios de salud, y población con poca educación para el cuidado del niño y migrante.

Conclusiones: No se encontró un impacto positivo entre el PAN y la disminución de la prevalencia de desnutrición crónica a nivel de la DIRESA Callao 2009-2015. Los factores cualitativos que influyen en el PAN estuvieron relacionados a procesos y a la organización de la DIRESA Callao, a excepción de la población que corresponde a un factor externo.

Palabras clave: Desnutrición; Programas nacionales de salud; Evaluación del impacto en la salud (Fuente : DeCS BIREME).

\section{The Articulated Nutritional Program and its impact on chronic malnutrition in children under five years of age. Dirección Regional de Salud Callao, 2009-2015}

\section{ABSTRACT}

Objective: To determine the impact of the Articulated Nutritional Program (PAN) on chronic malnutrition in children under five years of age, its correlation with the PAN's budget execution, and the prevalence of chronic malnutrition at the Dirección Regional de Salud (DIRESA) Callao, 2009-2015.

Materials and methods: Aquantitative, qualitative, observational, ecological, correlational, longitudinal and retrospective study was carried out. The Pearson correlation coefficient was determined in the quantitative analysis. Qualitative data was obtained from in-depth interviews with the support of a semi-structured guide.

Results: There is no relationship between the PAN's Modified Institutional Budget (PIM) and the prevalence of chronic malnutrition $(p=0.92)$. There is no relationship between the budget execution of the PIM and the prevalence of chronic malnutrition $(p=0.355)$. The factors that influenced the execution of the PAN are untimely budget, delayed implementation of the PAN, difficulties in the organization of health services, and population with little education on child and migrant care.

Conclusions: No positive impact was found between the PAN and the decrease in the prevalence of chronic malnutrition at the DIRESA Callao between 2009 and 2015. The qualitative factors that influence the PAN were related to processes and the organization of the DIRESA Callao, with the exception of the population corresponding to an external factor.

Keywords: Malnutrition; National health programs; Health impact assessment (Source: MeSH NLM).

\footnotetext{
1 Universidad de San Martín de Porres, Facultad de Medicina Humana. Lima, Perú.

2 Centro de Investigación en Salud Pública. Lima, Perú.

a Médico Cirujano, Especialista de Gestión en Salud. Magíster en Salud Pública y Gestión de Sistemas de Salud.

b Médico Cirujano, Magíster en Salud Pública, Máster en Investigación de Salud.

c Médico Cirujano, Especialista en Epidemiología, Magíster en Gobierno y Gerencia en Salud.

* Autor corresponsal
} 


\section{INTRODUCCIÓN}

El Programa Articulado Nutricional (PAN) fue creado en el año 2008 para combatir la desnutrición crónica en menores de 5 años y pertenece a la estrategia de gestión pública denominada presupuesto por resultados ${ }^{(1-3)}$.

El citado programa considera productos orientados hacia el control de los determinantes que intervienen en la desnutrición, uno de ellos es el mejorar la alimentación y nutrición ${ }^{(2)}$.

Cruzado describió que el Programa Articulado Nutricional tiene como objetivo la reducción de la prevalencia de la desnutrición crónica infantil, que incluye una serie de actividades y productos; y concluyó que el control de crecimiento y desarrollo del niño y la vacunación completa son productos con la mayor cobertura y calidad a nivel del programa ${ }^{(4)}$.

Asimismo, describió que todo programa presupuestal necesita ser evaluado, tanto a nivel de la ejecución financiera y de metas físicas, y que es necesaria la realización del análisis de los avances anuales para así identificar el riesgo de ejecución financiera inadecuada (4); y, en ese sentido, el Ministerio de Economía y Finanzas señaló que el Programa Articulado Nutricional, desde 2013, se evalúa mediante informes o reportes mensuales, que se hace efectiva a través del porcentaje ejecutado del Presupuesto Institucional Modificado ${ }^{(5)}$.

La Organización Panamericana de la Salud describió a la desnutrición crónica como un problema grave de Salud Pública, debido a que representa el reflejo de la pobreza y la inequidad para el acceso a los servicios básicos requeridos por millones de personas en América Latina y El Caribe ${ }^{(6)}$.

La Organización Mundial de la Salud señaló que el retraso del crecimiento se presenta en una niña o niño con una talla insuficiente con relación a su edad, lo cual se debería a su condición de desnutrición crónica o recurrente, un problema de salud pública que impide que los niños desarrollen plenamente su potencial físico y cognitivo y que guarda estrecha relación con los determinantes sociales ${ }^{(7)}$; condición que también se describió en el estudio de Aldana ${ }^{(8)}$.

Los datos del Instituto Nacional de Estadística e Informática y del Centro Nacional de Alimentación y Nutrición del Instituto Nacional de Salud muestran que la desnutrición crónica se encontró en 7,3\% ${ }^{(9)}, 5,4 \%{ }^{(10)}$ y $8,9 \%{ }^{(11)}$; es decir, afectó a un total de 74872,77135 y 78006 infantes entre 2000, 2005 y 2007, respectivamente.

Según el Plan Estratégico Institucional de la Dirección Regional de Salud Callao, esta institución es una dependencia técnica y administrativa del Gobierno Regional del Callao, que consigna como uno de sus objetivos estratégicos la disminución de la desnutrición crónica infantil ${ }^{(12)}$.

El informe de actividades del Plan Operativo Anual de la Dirección Regional de Salud Callao de 2013, indicó que el Programa Articulado Nutricional alcanzó una ejecución de gasto de $99,98 \%$ en relación a lo asignado en el Presupuesto Institucional Modificado ${ }^{(13)}$; asimismo, en su informe de Evaluación del Plan Operativo Institucional de 2014 se indicó que el citado programa alcanzó una ejecución de gasto de S/19 335176 (99,44\%), y mostró el cumplimiento de reducción de la desnutrición crónica en un $97,0 \%$ (7,9\%) en relación a la meta esperada $(7,65 \%)^{(14)}$.

El objetivo del presente estudio fue determinar el impacto del Programa Articulado Nutricional sobre la desnutrición crónica en menores de cinco años en la Dirección Regional de Salud Callao, 2009-2015.

\section{MATERIALES Y MÉTODOS}

\section{Diseño y población de estudio}

Se realizó un estudio tipo cuanticualitativo, observacional ecológico, correlacional, longitudinal y retrospectivo. La población para la metodología cualitativa fueron todos los individuos que trabajaron en el Programa Articulado Nutricional (PAN) de la Dirección Regional de Salud Callao (DIRESA Callao) y que estuvieron dispuestos a participar. Se trabajó con fuentes secundarias, base de datos del PAN a nivel de la DIRESA Callao, consulta amigable del Ministerio de Economía y Finanzas (MEF), Instituto Nacional de Estadística e Informática (INEI) y Encuesta Demográfica y de Salud Familiar (ENDES), y se tomaron en cuenta el total de los datos según las hipótesis a comprobar.

Para la obtención de datos de la investigación cuantitativa, se utilizaron fichas de recolección de datos, en las cuales se consignaron los datos referidos al Presupuesto Institucional Modificado (PIM) del PAN y la ejecución del mismo, así como los datos de prevalencia de la desnutrición crónica.

Para obtener datos cualitativos, se aplicó una entrevista minuciosa a los participantes en las actividades del programa presupuestal. La entrevista se apoyó en una guía semiestructurada de cinco preguntas elaboradas específicamente para este estudio que fueron las siguientes: ¿cuáles son los factores que influyen sobre la ejecución del PAN?, ¿qué dificultades han presentado para la implementación del PAN en la DIRESA Callao?, ¿cuáles son las fortalezas del PAN?, ¿cuáles son las oportunidades que se han presentado con el PAN? y ¿por qué cree que no hay una mayor reducción de la desnutrición crónica en la población de la jurisdicción de la DIRESA Callao?

Con base en las respuestas obtenidas en la entrevista, 
se categorizó y sintetizó la información cualitativa en un modelo explicativo de la prevalencia de desnutrición crónica, que se resumieron en posibles explicaciones de los resultados en relación al impacto del Programa Articulado Nutricional sobre la Desnutrición Crónica en menores de cinco años.

Los criterios de inclusión fueron los datos del Programa Articulado Nutricional (PAN) a nivel de la DIRESA Callao, de 2009 a 2015 (para la investigación cuantitativa), y el personal de salud que trabajó en el PAN de la DIRESA Callao, y que estuvieron dispuestos a participar (para la investigación cualitativa). Como criterios de exclusión se consideraron a los programas articulados nutricionales a nivel de otras DIRESAS, otros programas presupuestales, y el personal de salud de otras dependencias o que no deseaban participar.

\section{Variables y mediciones}

Las variables del presente estudio fueron el Programa Articulado nutricional (PAN), dentro del cual se consideró la ejecución presupuestal del Presupuesto Institucional Modificado (PIM) asignado al programa, y la desnutrición crónica infantil expresada a nivel de prevalencia.

Los datos referidos al PIM del PAN, así como la ejecución presupuestal del mismo, se obtuvieron de la consulta amigable del MEF; y para la obtención de la tasa de desnutrición crónica, se tuvieron en cuenta los datos reportados por ENDES e INEI en relación a la región Callao y datos proporcionados por la DIRESA Callao.

Para la investigación cualitativa, se realizó una entrevista a profundidad a los responsables de áreas de la DIRESA Callao, involucradas con las actividades del Programa Articulado
Nutricional (PAN), con base a lo cual se categorizó y sintetizó la información cualitativa que se resumió en posibles explicaciones de los resultados del impacto del PAN sobre la desnutrición crónica en menores de cinco años.

\section{Análisis estadístico}

Una vez obtenidos los datos cuantitativos se procedió con el registro y procesamiento de los mismos. En el programa estadístico SPSS se aplicó el coeficiente de correlación de Pearson, método estadístico apropiado para ver correlaciones entre variables de escala de razón. Asimismo, se revisaron los datos de desnutrición crónica de algunos años previos al 2009, así como del período 2009 y 2015 y, con base en esta información, se analizó, de manera indirecta, el impacto según los resultados encontrados.

\section{Consideraciones éticas}

La obtención de la información contó con la aprobación de la DIRESA Callao, y no existieron conflictos éticos para su aplicación.

\section{RESULTADOS}

Se encontró que existe un incremento del Presupuesto Institucional Modificado (PIM) del Programa Articulado nutricional (PAN), que se observa también a nivel de sus productos (niños con CRED completo y niños con vacuna completa). Se determinó la correlación de Pearson entre el PIM del PAN, sus productos (niños con CRED completo y niños con vacuna completa), y la prevalencia de desnutrición crónica en menores de cinco años Dirección Regional de Salud Callao 2009-2015, y no se encontró evidencia para concluir que existe una relación entre dichas variables $(p=0,921 ; 0,644$ y 0,247 ; respectivamente) (Tabla 1$)$.

Tabla 1. Correlación entre el PIM del PAN (sus productos Niños con CRED completo y Niños con vacuna completa) y el porcentaje desnutrición crónica en menores de cinco años Dirección Regional de Salud (DIRESA) Callao 2009-2015

\begin{tabular}{|c|c|c|c|c|c|c|c|c|c|}
\hline \multicolumn{2}{|c|}{$\begin{array}{l}\text { Años DIRESA } \\
\text { CALLAO }\end{array}$} & \multicolumn{2}{|c|}{$\begin{array}{l}\text { Programa Articulado } \\
\text { Nacional (PAN) }\end{array}$} & \multicolumn{3}{|c|}{$\begin{array}{l}\text { Producto del PAN: Niños } \\
\text { con CRED completo }\end{array}$} & \multicolumn{3}{|c|}{$\begin{array}{l}\text { Producto del PAN: Niños } \\
\text { con vacuna completa }\end{array}$} \\
\hline & $\begin{array}{l}\text { PIM } \\
\text { asignado } \\
\text { al PAN }\end{array}$ & $\begin{array}{l}\% \text { de } \\
\text { nutrición } \\
\text { crónica }\end{array}$ & $\begin{array}{l}\text { Coeficiente } \\
\text { de correlación } \\
\text { de Pearson }\end{array}$ & $\begin{array}{l}\text { PIM asignado al } \\
\text { producto del } \\
\text { PAN }\end{array}$ & $\begin{array}{l}\% \text { de } \\
\text { nutrición } \\
\text { crónica }\end{array}$ & $\begin{array}{l}\text { Coeficiente de } \\
\text { correlación } \\
\text { de Pearson }\end{array}$ & $\begin{array}{l}\text { PIM del } \\
\text { PAN }\end{array}$ & $\begin{array}{l}\% \text { de } \\
\text { nutrición } \\
\text { crónica }\end{array}$ & $\begin{array}{c}\text { Coeficiente } \\
\text { de correlación } \\
\text { de Programa } \\
\text { Articulado Nacional }\end{array}$ \\
\hline 2009 & S/ 6739062 & 8,0 & \multirow{7}{*}{$\begin{array}{l}r p=0,046 \\
p=0,921\end{array}$} & S/ 577070 & 8,0 & \multirow{7}{*}{$\begin{array}{l}r p=0,214 \\
p=0,644\end{array}$} & S/ 940774 & 8,0 & \multirow{7}{*}{$\begin{array}{l}r p=0,506 \\
p=0,247\end{array}$} \\
\hline 2010 & S/ 6238469 & 6,0 & & S/ 769196 & 6,0 & & S/1 290624 & 6,0 & \\
\hline 2011 & S/10 880711 & 9,8 & & S/1 518047 & 9,8 & & S/ 2731829 & 9,8 & \\
\hline 2012 & S/ 16737749 & 9,1 & & S/1 647289 & 9,1 & & S/ 4884917 & 9,1 & \\
\hline 2013 & S/ 16262272 & 8,5 & & S/ 2864920 & 8,5 & & S/ 3613831 & 8,5 & \\
\hline 2014 & S/ 19464071 & 7,6 & & S/ 4386327 & 7,6 & & S/ 2918819 & 7,6 & \\
\hline 2015 & S/ 23537438 & 7,0 & & S/ 6006961 & 7,0 & & S/ 3116476 & 7,0 & \\
\hline
\end{tabular}

Fuente: *Página amigable del MEF. **Datos proporcionados por la DIRESA Callao. 
Se observó un incremento del presupuesto asignado a Recursos Humanos de S/5 435325 soles, en el año 2009, a S/13 222238 soles en el año 2015; sin embargo, el porcentaje de asignación presupuestal en recursos humanos se encontró en más del $80 \%$, y es menor a partir del año 2012 (>70 \%), y presentó su valor más bajo en el año 2015 (56,2\%) (Tabla 2).

Tabla 2. Distribución del PIM del PAN en Recursos Humanos DIRESA Callao 2009-2015

\begin{tabular}{|c|c|c|c|c|c|c|c|}
\hline \multicolumn{8}{|c|}{ Presupuesto del Programa Articulado Nutricional, DIRESA CALLAO, 2009-2015 } \\
\hline Años & 2009 & 2010 & 2011 & 2012 & 2013 & 2014 & 2015 \\
\hline Presupuesto (PIM) & $S / 6739,062$ & $S / 6238,469$ & S/ 10880,711 & $S / 16737,749$ & S/ 16262,272 & S/ 19464,071 & S/ 23537,438 \\
\hline Personal y obligaciones & S/ 5155,669 & S/ 3691.298 & S/ 6578,592 & S/ 6115,957 & S/ 7677,179 & S/ 9509,858 & S/ 10379,570 \\
\hline CAS & S/ 279,656 & S/ 1473.924 & S/ 2341,483 & S/ 6222,319 & $S / 4467,126$ & $S / 3605,305$ & $S / 2842,668$ \\
\hline Total de PIM en RR. HH. & S/ 5435,325 & S/ 5165.222 & S/ 8920,075 & $S / 12338,276$ & S/ 12144,305 & $S / 13115,163$ & $S / 13222,238$ \\
\hline \% de PIM en RR. HH. & 80,7 & 82,8 & 82,0 & 73,7 & 74,7 & 67,4 & 56,2 \\
\hline Total de PIM en otros & S/ 1303,737 & S/ 1073,247 & S/ 1960,636 & S/ 4399,473 & S/ 4117,967 & S/ 6348,908 & S/ 10315,200 \\
\hline$\%$ de PIM en otros & 19,3 & 17,2 & 18,0 & 26,3 & 25,3 & 32,6 & 43,8 \\
\hline
\end{tabular}

Fuente: Consulta amigable del MEF.

Se observó que a nivel de la DIRESA Callao existe una ejecución presupuestal del PAN mayor al $90 \%$, entre 2009-2015. Se determinó la correlación de Pearson entre la ejecución presupuestal de PAN, sus productos Niños con CRED completo y Niños con vacuna completa, y la prevalencia de desnutrición crónica en menores de cinco años Dirección Regional de Salud Callao 2009-2015, y no se encontró evidencia para concluir que existe una relación entre dichas variables $(p=0,355 ; 0,821$ y 0,239 ; respectivamente) (Tabla 3 ).

Tabla 3. Correlación entre la ejecución presupuestal del PAN, sus productos Niños con CRED completo y Niños con vacuna completa, y el porcentaje desnutrición crónica en menores de cinco años DIRESA Callao 2009-2015

\begin{tabular}{|c|c|c|c|c|c|c|c|c|c|}
\hline \multicolumn{2}{|c|}{$\begin{array}{l}\text { Años DIRESA } \\
\text { CALLAO }\end{array}$} & \multicolumn{2}{|c|}{$\begin{array}{l}\text { Programa Articulado } \\
\text { Nacional (PAN) }\end{array}$} & \multicolumn{3}{|c|}{$\begin{array}{l}\text { Producto del PAN: Niños } \\
\text { con CRED completo }\end{array}$} & \multicolumn{3}{|c|}{$\begin{array}{l}\text { Producto del PAN: Niños } \\
\text { con vacuna completa }\end{array}$} \\
\hline & $\begin{array}{l}\text { \% de } \\
\text { presupuestal } \\
\text { del PAN }\end{array}$ & $\begin{array}{l}\text { \% de } \\
\text { nutrición } \\
\text { crónica }\end{array}$ & $\begin{array}{l}\text { Coeficiente } \\
\text { correlación } \\
\text { de Pearson }\end{array}$ & $\begin{array}{l}\text { \% de ejecución } \\
\text { presupuestal } \\
\text { del PAN }\end{array}$ & $\begin{array}{l}\text { \% de } \\
\text { nutrición } \\
\text { crónica }\end{array}$ & $\begin{array}{l}\text { Coeficiente de } \\
\text { correlación } \\
\text { de Pearson }\end{array}$ & $\begin{array}{l}\text { \% de ejecución } \\
\text { presupuestal } \\
\text { del PAN }\end{array}$ & $\begin{array}{l}\text { \% de } \\
\text { nutrición } \\
\text { crónica }\end{array}$ & $\begin{array}{l}\text { Coeficiente } \\
\text { de correlación } \\
\text { de Pearson }\end{array}$ \\
\hline 2009 & 96,4 & 8,0 & \multirow{7}{*}{$\begin{array}{l}r p=0,415 \\
p=0,355\end{array}$} & 87,1 & 8,0 & \multirow{7}{*}{$\begin{array}{l}r p=0,106 \\
p=0,821\end{array}$} & 98,8 & 8,0 & \multirow{7}{*}{$\begin{array}{l}r p=0,513 \\
p=0,239\end{array}$} \\
\hline 2010 & 97,5 & 6,0 & & 95,2 & 6,0 & & 94,6 & 6,0 & \\
\hline 2011 & 95,7 & 9,8 & & 91,1 & 9,8 & & 100,0 & 9,8 & \\
\hline 2012 & 95,6 & 9,1 & & 82,4 & 9,1 & & 95,7 & 9,1 & \\
\hline 2013 & 99,9 & 8,5 & & 100,0 & 8,5 & & 99,7 & 8,5 & \\
\hline 2014 & 99,4 & 7,6 & & 99,9 & 7,6 & & 99,7 & 7,6 & \\
\hline 2015 & 98,1 & 7,0 & & 98,9 & 7,0 & & 97,6 & 7,0 & \\
\hline
\end{tabular}

Fuente: *Consulta amigable del MEF. **Datos proporcionados por la DIRESA Callao. 
Se observó que la desnutrición crónica en el 2009 se encontró en un $8 \%$, es decir, que afectó a 6292 infantes a nivel de la región Callao; y llega a disminuir a un $6 \%$ en 2010 (4728 niños); sin embargo, se observó un incremento a un $9,8 \%$ en 2011 (7720 niños) y, a partir de dicho año, se observó una disminución en el porcentaje de desnutrición crónica infantil, tal es así, que en 2015 se encontró en un 7 \% (5454 niños).
Asimismo, los datos del porcentaje de desnutrición crónica infantil de la región Callao, 7,3 \% (5466 niños), 5,4\% (4165 niños) y $8,9 \%$ (6943 niños), correspondiente a los años 2000, 2005 y 2007, respectivamente. Estos datos se encontraron cercanos a los porcentajes presentados en el período 2009-2015; con una diferencia de solo un 0,3\% entre 2000 y 2015 , pese a que partir del 2009 ya se contaba con el PAN, tal como se observa en la tabla 4.

Tabla 4. Población menor de cinco años con desnutrición crónica DIRESA Callao 2000, 2005, 2007, 2009-2015

\begin{tabular}{|cccc|}
\hline Año & $\begin{array}{c}\text { Población total niños } \\
\text { menores } 5 \text { años }\left(^{*}\right)\end{array}$ & $\begin{array}{c}\text { \% desnutrición crónica } \\
\text { en menores de } 5 \text { años }\end{array}$ & $\begin{array}{c}\text { Total de niños con } \\
\text { desnutrición crónica }\end{array}$ \\
\hline 2000 & 74872 & $7,3\left(^{* *}\right)$ & 5466 \\
\hline 2005 & 77135 & $5,4\left(^{* *}\right)$ & 4165 \\
\hline 2017 & 78006 & $8,9\left(^{* *}\right)$ & 6943 \\
\hline 2009 & 78654 & $8,0\left(^{* * *}\right)$ & 6292 \\
\hline 2010 & 78807 & $6,0\left(^{* * *}\right)$ & 4728 \\
\hline 2011 & 78778 & $9,8\left(^{* * *}\right)$ & 7720 \\
\hline 2012 & 78590 & $9,1\left(^{* * *}\right)$ & 7152 \\
\hline 2013 & 78328 & $8,5\left(^{* * *}\right)$ & 6658 \\
\hline 2014 & 78076 & $7,6\left(^{* * *}\right)$ & 5934 \\
\hline 2015 & 77921 & $7,0\left(^{* * *}\right)$ & 5454 \\
\hline
\end{tabular}

Fuente: $\left({ }^{*}\right)$ INEI. $\left.{ }^{* *}\right)$ Encuesta ENDES. ${ }^{(* *)}$ DIRESA Callao.

En la tabla 5 se puede observar que la población con desnutrición crónica se concentra mayormente en los quintiles segundo, intermedio y cuarto, y es menor a nivel del primer y quinto quintiles de pobreza. Asimismo, se observa una disminución entre los años 2009 y 2013, con un ligero incremento en el año 2015 con respecto al año 2014. Finalmente, es importante tener en cuenta que a nivel del Callao es mínima la población a nivel del quintil inferior.

Tabla 5. Población de niños menores con desnutrición crónica en niños menores de cinco años con desnutrición crónica, según quintiles de pobreza, región Callao, 2009-2015

\begin{tabular}{|c|c|c|c|c|c|c|c|c|c|c|}
\hline \multirow[b]{2}{*}{ Años } & \multicolumn{2}{|c|}{ Quintil inferior } & \multicolumn{2}{|c|}{ Segundo quintil } & \multicolumn{2}{|c|}{ Quintil intermedio } & \multicolumn{2}{|c|}{ Cuarto quintil } & \multicolumn{2}{|c|}{ Quintil superior } \\
\hline & $\begin{array}{c}\% \text { DC } \\
\left({ }^{* *}\right)\end{array}$ & $\begin{array}{l}\text { Población } \\
\text { con DC }\left(^{*}\right)\end{array}$ & $\begin{array}{c}\% \mathrm{DC} \\
(* *)\end{array}$ & $\begin{array}{l}\text { Población } \\
\text { con DC }\left({ }^{*}\right)\end{array}$ & $\begin{array}{c}\% \text { DC } \\
(* *)\end{array}$ & $\begin{array}{l}\text { Población } \\
\text { con DC }\left(^{*}\right)\end{array}$ & $\begin{array}{c}\% \text { DC } \\
(* *)\end{array}$ & $\begin{array}{l}\text { Población } \\
\text { con DC (*) }\end{array}$ & $\begin{array}{c}\% \mathrm{DC} \\
(* *)\end{array}$ & $\begin{array}{l}\text { Población } \\
\text { con DC }\left(^{*}\right)\end{array}$ \\
\hline 2009 & 45,3 & 107 & 29,7 & 1,028 & 14,1 & 2,107 & 10,1 & 2,423 & 4,2 & 198 \\
\hline 2010 & 44,0 & 277 & 28,6 & 1,397 & 15,4 & 2,476 & 7,3 & 1,703 & 5,2 & 279 \\
\hline 2011 & 43,8 & 138 & 22,3 & 1,072 & 9,6 & 1,513 & 6,5 & 1,516 & 2,8 & 196 \\
\hline 2012 & 38,8 & 91 & 20,1 & 0,948 & 11,5 & 1,672 & 5,4 & 1,282 & 3,1 & 205 \\
\hline 2013 & 37,6 & 29 & 21,2 & 1,129 & 8,7 & 1,377 & 4,1 & 944 & 2,3 & 326 \\
\hline 2014 & 34,0 & 106 & 14,1 & 0,980 & 8,3 & 1,322 & 4,0 & 956 & 3,7 & 901 \\
\hline 2015 & 31,6 & 172 & 15,7 & 1,028 & 3,1 & 437 & 5,5 & 1,706 & 2,8 & 908 \\
\hline
\end{tabular}

Fuente: Encuesta Nacional de Hogares (ENAHO) 2009-2015

$\left(^{*}\right)$ Estimación de la población con desnutrición crónica, teniendo en cuenta el total de la población estimada según porcentajes por quintiles de pobreza a nivel de Lima Metropolitana (ENAHO 2009-2015).

$\left.{ }^{* *}\right)$ \% DC: Porcentaje de desnutrición crónica. 
Por tanto, si consideramos que no se encontró un impacto positivo del PAN sobre la desnutrición crónica en menores de cinco años, ni correlación significativa tanto del PIM del PAN y de la ejecución del mismo con los porcentajes de desnutrición crónica; se desarrolló una investigación cualitativa, en la cual se encontraron algunas posibles explicaciones de los resultados de la investigación cuantitativa, las mismas que se presentan en la tabla 6 .

Tabla 6. Posibles explicaciones de la ausencia de correlación entre la ejecución del Programa Articulado Nutricional y la prevalencia de desnutrición crónica en menores de cinco años DIRESA Callao 2009-2015

\begin{tabular}{|c|c|c|}
\hline N. ${ }^{\circ}$ & Posible explicación & Especificaciones \\
\hline 1 & $\begin{array}{l}\text { Presupuesto } \\
\text { inoportuno }\end{array}$ & $\begin{array}{l}\text { Por desvío de la cadena presupuestaria programada, a pesar del registro } \\
\text { de ejecución en actividades del PAN. } \\
\text { Por demora en la aprobación del presupuesto, lo que retrasa la ejecución } \\
\text { presupuestal. } \\
\text { Por demora en la obtención de materiales para la ejecución de } \\
\text { actividades del PAN. } \\
\text { Porque se prioriza la elaboración de material de difusión y no se contrata } \\
\text { suficiente personal. }\end{array}$ \\
\hline 2 & $\begin{array}{l}\text { Retraso en la } \\
\text { implementación } \\
\text { del Programa Articulado } \\
\text { Nutricional (PAN) }\end{array}$ & $\begin{array}{l}\text { Por demora en compra de bienes y servicios que se necesitan en las } \\
\text { actividades del PAN, lo cual influye en el cumplimiento de metas e } \\
\text { indicadores. } \\
\text { Por cambios constantes en las propuestas y definiciones operacionales } \\
\text { del PAN. } \\
\text { Por los recursos humanos limitados para el cumplimiento de metas. } \\
\text { Por demora en el proceso logístico para la obtención de materiales. } \\
\text { Por demora en el proceso logístico para la obtención de materiales. } \\
\text { Por pocas actividades extramurales. } \\
\text { Por el trabajo de campo limitado, debido a la delincuencia. } \\
\text { Por la limitada capacitación a los responsables del PAN, respecto a las } \\
\text { definiciones actuales del PAN. }\end{array}$ \\
\hline 3 & $\begin{array}{l}\text { Dificultades en la } \\
\text { organización de los } \\
\text { servicios de salud }\end{array}$ & $\begin{array}{l}\text { Porque hay campañas de salud, pero sobre todo a nivel intramural. } \\
\text { Porque se realizan pocos trabajos con juntas vecinales que comprometen } \\
\text { a la misma comunidad. "Es poco, pero se hace". } \\
\text { Por el apoyo de ONG, solo por tiempo limitado. } \\
\text { Por la falta de recursos humanos, para realizar las consejerías en } \\
\text { nutrición. } \\
\text { Porque se requiere mayor asignación de presupuesto. } \\
\text { Porque se requiere mayor capacitación del personal involucrado con las } \\
\text { actividades del PAN. }\end{array}$ \\
\hline 4 & Población & $\begin{array}{l}\text { Porque la educación de la madre no permite el buen entendimiento de } \\
\text { la información que se brinda respecto al cuidado del niño menor de } 3 \\
\text { años. } \\
\text { Porque existen aspectos culturales de la población, generan dificultad } \\
\text { para acceder a los controles de los niños. } \\
\text { Por la presencia de población inmigrante (de otras regiones del país), } \\
\text { con dificultad para poner en práctica las recomendaciones nutricionales, } \\
\text { esto en relación a los alimentos que se consumen según costumbres. }\end{array}$ \\
\hline \multicolumn{3}{|c|}{$\begin{array}{l}\text { Dentro de las preguntas de la entrevista, se consideró el tema de fortalezas el PAN y se obtuvieron las siguientes } \\
\text { respuestas: ordenamiento del gasto, contratación de personal para realizar actividades del PAN e interés de las } \\
\text { autoridades al tratarse de un tema de prioridad nacional. }\end{array}$} \\
\hline
\end{tabular}




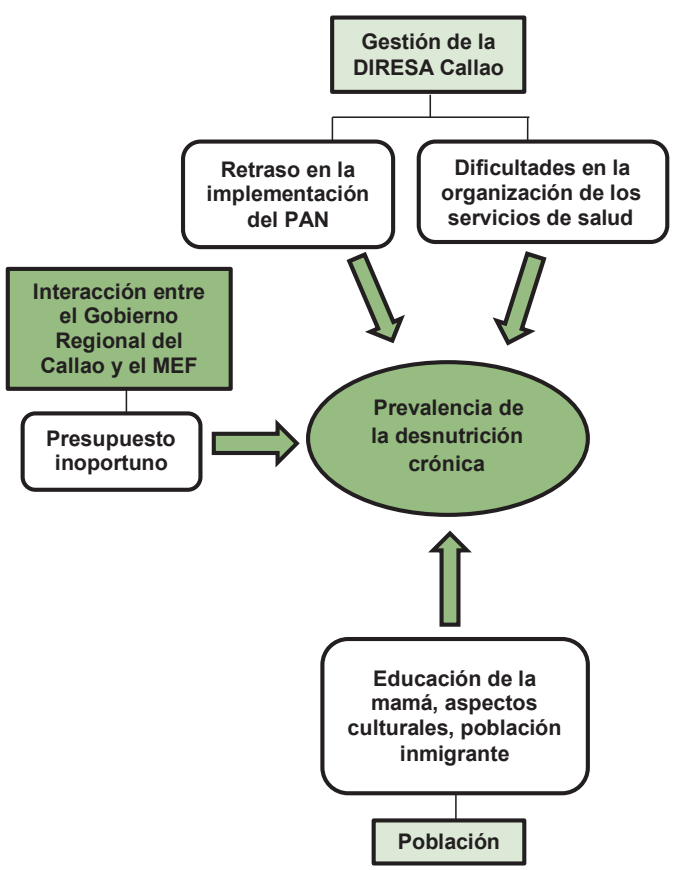

Figura 1. Posibles explicaciones de la prevalencia de la desnutrición crónica en menores de cinco años a nivel de la DIRESA Callao $2009-2015$

\section{DISCUSIÓN}

En cuanto a la asignación del PIM a nivel de la DIRESA Callao, para las actividades del Programa Articulado Nutricional (PAN), se observó un incremento de S/ 6739062 soles en 2009 a S/ 23537438 soles en 2015, lo cual concuerda con la publicación de CEPAL ${ }^{(15)}$, en relación al presupuesto por resultados.

Asimismo, se encontró una prevalencia de desnutrición crónica menor al 10,0\%, entre 2009 y 2015 , en menores de cinco años a nivel de la DIRESA Callao; y se encontraron los menores porcentajes en $2010(6,0 \%)$ y $2015(7,0 \%)$, esto pese a que el presupuesto en 2010 fue aproximadamente $73 \%$ menor al presupuesto de 2015 . Este resultado guardó cierta relación con lo señalado en el documento de CEPAL y Unicef (16), respecto a que la desnutrición crónica infantil a nivel de Lima Metropolitana solo ha disminuido en un $3,2 \%$.

En relación a la población con desnutrición crónica infantil en la región Callao, en 2015, se concentró en un mayor número a nivel del segundo quintil (1028), quintil intermedio (437) y cuarto quintil (1706); resultado que se correlaciona con el documento de CEPAL y Unicef que reportó un aumento de la desnutrición crónica infantil en los mismos quintiles de pobreza ${ }^{(16)}$.

El presente estudio observó que la ejecución presupuestal del PAN fue mayor al $90 \%$ en el período de tiempo analizado, y llegó a un $99,9 \%$ en 2013 y $98,15 \%$, en 2015 ; resultado que guarda relación con el estudio de USAID (17), el cual indica que el PAN fue uno de los programas con mejor ejecución presupuestal.

Con respecto a uno de los resultados de la correlación, se encontró que no existe una relación entre el PIM del PAN y el porcentaje de desnutrición crónica $(\mathrm{p}=0,921)$, en la DIRESA Callao, periodo 2009 - 2015, y resultado similar se encontró, en la correlación realizada entre el PIM de los productos del PAN (niños con CRED completo, niños con vacuna completa) y el porcentaje de desnutrición crónica infantil ( $p=0,644$ y 0,247 respectivamente). Esto podría estar relacionado con lo descrito en el Resumen Ejecutivo $2012^{(18)}$, que señala la no correspondencia entre la presentación de la desnutrición crónica y la asignación del presupuesto.

En lo correspondiente a otro de los resultados de correlación, se encontró que no existe evidencia para concluir que existe una relación entre las variables PAN (ejecución presupuestal) y desnutrición crónica infantil, en la DIRESA Callao, periodo 2009 - 2015 ( $p=0,742)$, lo cual podría indicar que existen otros factores distintos a la ejecución del presupuesto, factores que deben ser investigados para tenerse en cuenta en el proceso de priorización, realización y evaluación de las actividades del PAN; resultado relacionado con lo descrito en 
el Resumen Ejecutivo $2012{ }^{(18)}$, el cual señala la no correspondencia entre la presentación de la desnutrición crónica y la asignación del presupuesto.

Cabe precisar que la correlación realizada, a nivel del PIM y ejecución presupuestal de los productos más representativos del PAN (niños con CRED completo, niños con vacuna completa), y la prevalencia de desnutrición crónica infantil, fue realizada a nivel poblacional (no individual), hecho que no permite comparar los resultados del presente estudio con los resultados de Cruzado ${ }^{(4)}$, que muestra una relación positiva entre la creación del PAN y la reducción de la desnutrición crónica infantil pero no permite inferir en una relación de causalidad, aspecto que es importante conocer para el ajuste de estrategias de intervención por parte de los tomadores de decisiones, como por ejemplo en las propuestas de interrelación regional y local que se señala en el documento de la Mesa de Concertación de la Región Junín (19).

La prevalencia de desnutrición crónica, entre 2009 y 2015, presentó una variación mínima entre los años 2000 $(7,3 \%)$ y 2015 (7,0\%); es decir, de solo $0,3 \%$, con lo cual, indirectamente, no se observaría un impacto positivo del PAN sobre la prevalencia de desnutrición crónica infantil en menores de cinco años a nivel de la DIRESA Callao, pese a que a partir del año 2009 se contaba con presupuesto asignado para el PAN, a diferencia del año 2000 en el que aún no se contaba con el PAN. Este resultado requiere mayor análisis para la identificación de las causas que podrían estar influyendo en la leve disminución de la desnutrición crónica a nivel de la DIRESA Callao, y en donde valdría la pena evaluar los determinantes sociales en cada uno de los productos abordados mediante el PAN; lo cual está relacionado con lo descrito en el estudio Desnutrición infantil en menores de cinco años en Perú ${ }^{(20)}$, referente a la necesidad de hacer hincapié en los factores determinantes asociados a la desnutrición crónica, para disminuir las tasas de desnutrición crónica.

El presente estudio, mediante la investigación cualitativa, llegó a obtener posibles explicaciones a la ausencia de correlación entre las variables del estudio:

Presupuesto inoportuno: desvío de la cadena presupuestaria programada, demora en la aprobación del presupuesto, demora en la obtención de materiales para la ejecución de actividades del PAN, prioriza la elaboración de material de difusión y no se contrata suficiente personal; aspectos que podrían estar relacionados con la menor realización de actividades de monitoreo, supervisión, evaluación y control del programa articulado nutricional; tal como se describe en el Resumen Ejecutivo del MEF ${ }^{(5)}$; el cual señala que el menor avance de ejecución se observó en acciones de monitoreo, supervisión, evaluación y control del PAN.
Retraso en la implementación del PAN: demora en compra de bienes y servicios requeridos en las actividades del PAN, cambios constantes en las definiciones operacionales del PAN, recursos humanos limitados para el cumplimiento de metas, demora en el proceso logístico para la obtención de materiales, limitadas actividades extramurales, limitada capacitación a los responsables del PAN. Respecto a este punto, el Informe Resumen Ejecutivo de 2012 (18), describió que el PAN debería enfocarse en intervenir a nivel de los determinantes que causan la desnutrición crónica infantil, centrados en los lugares que las originan, con una articulación de las intervenciones estatales y con actividades que deben llegar a cada persona beneficiaria, para alcanzar el impacto esperado.

Dificultades en la organización de los servicios de Salud: se realizan pocas actividades con juntas vecinales que comprometen a la misma comunidad; apoyo de ONG solo por tiempo limitado, falta de recursos humanos para realizar las consejerías en nutrición, se requiere mayor asignación de presupuesto y mayor capacitación del personal involucrado con las actividades del PAN. Respecto a este punto, el Informe Resumen Ejecutivo de $2012{ }^{(18)}$, describió que para eliminar la desnutrición crónica infantil se requiere de mayor asignación de presupuesto público, aunado a un mayor esfuerzo, compromiso y participación de todos los actores claves.

A nivel de la población: la educación de la madre no permite el buen entendimiento de la información que se brinda respecto al cuidado del niño menor de tres años, aspectos culturales de la población generan dificultad para acceder a los controles de los niños, población inmigrante con diferentes costumbres que les dificulta poner en práctica las recomendaciones nutricionales. Respecto a este punto, el estudio Desnutrición Crónica Infantil en el Perú (2) menciona que la educación de la madre tiene el impacto más fuerte en la desnutrición infantil.

La realización de un estudio mixto es una fortaleza de esta investigación porque permitió establecer la existencia o no de correlación de variables, y a partir de ello permitió encontrar posibles respuestas mediante datos cualitativos obtenidos de entrevista a actores involucrados en actividades del PAN en la DIRESA Callao. No obstante, en ese contexto, una debilidad del estudio se centra en la falta de incorporación de datos cualitativos desde el lado de la población beneficiaria del PAN.

Este tipo de estudio podría replicarse a nivel de otras unidades ejecutoras, ya que existe información disponible a nivel de la consulta amigable del MEF, y con lo cual se podría obtener mayor información respecto a los factores que pudieran estar influenciando o no sobre la implementación de actividades del PAN y la prevalencia 
de desnutrición crónica en nuestro país.

En conclusión, no se observa un impacto positivo del PAN sobre la prevalencia de desnutrición crónica infantil en menores de cinco años a nivel de la DIRESA Callao, entre 2009-2015.

Las posibles explicaciones de la leve disminución de la prevalencia de desnutrición crónica en menores de cinco años, a nivel de la DIRESA Callao, se relacionan con ciertos factores como presupuesto inoportuno, retraso en la implementación del PAN, dificultades en la organización de los servicios de salud (internos) y con factores de la población (externos) como la educación de la madre aspectos culturales, población proveniente de otras regiones del país.

Contribuciones de los autores: La Dra. Keila Shajian participó en el planteamiento del problema a investigar, elaboración de la metodología del estudio de investigación, desarrollo de la investigación y redacción de su contenido; el Dr. Raúl Carreño participó en el asesoramiento en el diseño del proyecto, apoyo en la metodología del estudio cuantitativo, análisis y presentación de tablas y el Dr. José Garay en la revisión del estudio y elaboración del modelo explicativo que consta como ilustración.

Fuentes de financiamiento: Este artículo ha sido financiado por los autores.

Conflicto de interés: Los autores declaran no tener ningún conflicto de interés en la publicación de este artículo.

\section{REFERENCIAS BIBLIOGRÁFICAS}

1. Sánchez-Abanto J. Evolución de la desnutrición crónica en menores de cinco años en el Perú. Rev Perú Med Exp Salud Publica. 2012; 29(3): 402-5.

2. Beltrán A, Seinfeld J. Desnutrición crónica infantil en el Perú: un problema persistente [Internet]. Centro de Investigación de la Universidad del Pacífico; 2009. Disponible en: http:// repositorio.up.edu.pe/bitstream/handle/11354/349/DD0914. pdf? sequence $=1$ \&isAllowed $=y$.

3. UNICEF. La desnutrición infantil. Causas, consecuencias y estrategias para su prevención y tratamiento [Internet]. 2011. Disponible en: https://www.unicef.es/sites/unicef.es/files/ Dossierdesnutricion.pdf

4. Cruzado V. Análisis del Impacto del programa articulado nutricional sobre la Desnutrición Crónica en niños menores de 5 años. DT01/2012 [Internet]. 2012. Disponible en: https://www.mef.gob. pe/contenidos/presu_publ/estudios/PAN_final_fusionado.pdf.

5. Ministerio de Economía y Finanzas. Reporte de Seguimiento de ejecución financiera. Programas Presupuestales con Enfoque de Resultados [Internet]. Lima; 2013. Disponible en: https://www. mef.gob.pe/contenidos/presu_publ/ppr/reporte_metas_fisicas_ financieras.pdf.

6. Alianza Panamericana por la Nutrición y el Desarrollo. Documento básico, premisas conceptuales y principios estratégicos. SINCO Editores S.A.C [Internet]. Lima; 2009. Disponible en: http://www. bvsde.paho.org/texcom/nutricion/alidoces.pdf.

7. Organización Mundial de la Salud. Malnutrición [Internet]. Disponible en: http://www.who.int/mediacentre/factsheets/ malnutrition/es/.

8. Aldana U. La desnutrición crónica en Lima Metropolitana. Revista Argumentos [Internet]. 2013. Disponible en: http:// revistaargumentos.iep.org.pe/wp_content/uploads/2014/04/ aldana_marzo2013.pdf.

9. Instituto Nacional de Estadística e Informática (INEI). Perú: Porcentaje de niñas y niños menores de cinco años de edad con desnutrición crónica, según característica seleccionada, 1991-92, 1996, 2000, 2009, 2014 y 2015 [Internet]. Disponible en: https:// www.inei.gob.pe/media/MenuRecursivo/publicaciones_digitales/ Est/Lib1211/cuadros/Cap_013.xls.

10. Instituto Nacional de Salud. Centro Nacional de Alimentación y Nutrición (CENAN). Prevalencia DNC 2005 0-59m [Internet]. Disponible en: http://www.ins.gob.pe/insvirtual/BiblioDig/ TREFS/LBdum.xls

11. Instituto Nacional de Estadística e Informática. Anexo № 1 Perú: población censada menor de 5 años, proporción de menores de 5 años con desnutrición crónica, según departamento, provincia y distrito, 2007 (Patrón OMS) [Internet]. Disponible en: https:// www.inei.gob.pe/media/MenuRecursivo/publicaciones_digitales/ Est/Lib0881/libro.pdf.

12. Dirección Regional de Salud Callao. Formulación del Plan Estratégico Institucional de la Dirección de Salud Callao 20132016 [Internet]. Disponible en: http://www.diresacallao.gob. pe/wdiresa/documentos/planeamiento/PEI_2013-2016_DIRESA_ Callao.pdf.

13. Dirección Regional de Salud Callao. Informe de Evaluación de Actividades del Plan Operativo Anual 2013 [Internet]. Disponible en: http://www.diresacallao.gob.pe/wdiresa/documentos/ planeamiento/EVA_POA_ANUAL_2013.pdf.

14. Dirección Regional de Salud Callao. Informe de Evaluación del Plan Operativo Anual 2014 [Internet]. Disponible en: http://www. diresacallao.gob.pe/wdiresa/documentos/planeamiento/EVA_ POA_ANUAL_2014.pdf.

15. Córdova F. Presupuesto por resultados: Un Instrumento Innovativo de Gestión Pública [Internet]. Disponible en: https://www.cepal.org/ ilpes/noticias/paginas/2/36342/presupuestoporresultados_F_ CORDOVA.pdf.

16. CEPAL, Unicef. La construcción de un sistema de protección social adecuado a la infancia en el Perú. Oportunidades y retos [Internet]. Disponible en: https://repositorio.cepal.org/ bitstream/handle/11362/43193/1/S1701141_es.pdf.

17. Sobrevilla A. Ejecución Presupuestal en Salud. Análisis del avance en la ejecución del presupuesto público en salud 2014 [Internet]. Disponible en: http://docplayer.es/66027222-Ejecucionpresupuestal-en-salud-analisis-del-avance-en-la-ejecucion-delpresupuesto-publico-en-salud-2014.html.

18. Inversión en la Infancia. Desnutrición crónica Infantil Cero en el 2016. Una meta alcanzable. El Infobarómetro de la Primera Infancia. Resumen Ejecutivo 2012 [Internet]. Disponible en: http://inversionenlainfancia.net/application/views/materiales/ UPLOAD/ARCHIVOS_DOCUMENTO/documento_documento_file/10_ desnutricion_resumen.pdf.

19. Mesa de Concertación para la Lucha contra la Pobreza. Comité Ejecutivo Regional Junín. Seguimiento Concertado al Acuerdo de Gobernabilidad para el Desarrollo Regional de Junín, 2015-2018 [Internet]. Disponible en: https://www.mesadeconcertacion.org. pe/publicaciones?page $=1$ 
20. Sobrino M, Gutiérrez C, Cunha A, Dávila M, Alarcón J. Desnutrición infantil en menores de cinco años en Perú: tendencias y factores determinantes. Rev Panam Salud Publica. 2014; 35(2): 104-12.

\section{Correspondencia:}

Keila Wendy Shajian Torres

Dirección: Calle Carlos Alayza y Roel 2392-

Dpto. 603 - Lince, Lima, Perú.

Teléfono: 980314072

Correo electrónico: mdkeilawendy@gmail.com
Recibido: 03 de junio de 2019

Evaluado: 13 de julio de 2019

Aprobado: 20 de agosto de 2019

(c) La revista. Publicado por Universidad de San Martín de Porres, Perú. (c) $\mathbf{B r}$ Licencia de Creative Commons Artículo en acceso abierto bajo términos de Licencia Creative Commons Atribución 4.0 Internacional. (http://creativecommons.org/licenses/by/4.0/)

ORCID iDs

Keila Wendy Shajian Torres

José Garay Uribe

(1) https: / / orcid.org/0000-0002-4446-0629

Ricardo Carreño Escobedo

(1) https: / / orcid.org/0000-0003-4052-3204

(1) https: / / orcid.org/0000-0003-3707-2764 